\title{
The Effect of Silver Nanoparticles on the Scavenger Receptor-Scara1 on Microglia
}

\author{
Sikorska Katarzyna, Grądzka Iwona , Wasyk Iwona \\ Institute of Nuclear Chemistry and Technology, Centre for Radiobiology and Biological Dosimetry \\ Dorodna 16, 03-195 Warsaw, Poland \\ k.sikorska@ichtj.waw.pl; i.gradzka@ichtj.waw.pl; iwonawasyk@gmail.com
}

\section{Extended Abstract}

Alzheimer's disease is one of the most prevalent neurodegenerative diseases in the world. The neuropathological hallmark of Alzheimer's disease is extracellular deposit of amyloid- $\beta$ (A $\beta)$ in the brain. Microglial cells are able to remove of $\mathrm{A} \beta$ aggregates by receptor-dependent endocytosis [1,3]. Nanotechnology is one of the fastest developing science discipline and nanoparticles (NPs), due to their strong absorption properties are widely used in industry, and also in medical diagnosis and treatment. It was documented that NPs can prevent the formation of Aß-aggregates whereby reducing their neurotoxicity and likely can impact on the Aß-uptake by microglia [2,4-7]. It is supposed that NPs can increase number of emerging phagocytosis bubbles and the $A \beta$ uptake due to the co-transport phenomenon, or in contrary reduce the number of lipid rafts available and therefore inhibit of $A \beta$ transport by some kind of competition. Moreover, by activating different paths to cell signaling, NPs can probably change the expression of the amyloid $\beta$-receptors on microglia cell membranes.

The goal of our study was to verify whether silver nanoparticles (AgNPs, $20 \mathrm{~nm}$, BSA coated) can change the ability of microglial scavenger receptor 1 (Scara1) for the $A ß$ (1-42) uptake and influence gene or protein expression of these receptors in mouse BV-2 cells.

The results from flow cytometry indicate that both $A \beta$ and AgNPs are taken up by microglial cells using the same receptor: AgNPs $(50 \mu \mathrm{g} / \mathrm{ml})$ can decrease the uptake of $A \beta$ by about $80 \%$ compared to the control group and Scara1 inhibitor (poliinosinic acid) diminish both AgNPs and Aß peptide uptake. Real-time PCR analysis showed that AgNPs did not change the Scara1 gene expression. The Western blotting (measuring the whole receptor content) revealed a slight decrease in the protein receptor level after treatment of cells with AgNPs $(50 \mu \mathrm{g} / \mathrm{ml})$. On the other hand, the content of the receptor on the cell surface, measured cytometrically, was greatly diminished in the presence of AgNPs.

In summary, AgNPs clearly blocked the receptor and so they may play rather disadvantageous role in $A \beta$ removal.

Results from the Project "The influence of nanoparticles on beta-amyloid removal by microglia cells". From National Science Centre, Project duration: 09. 2014-09.2017 Project ID:UMO-2013/11/N/NZ7/00415.

\section{References}

[1] L. M. Bekris et al., "Genetics of Alzheimer Disease," J Geriatr Psychiatry Neurol., vol. 23, no. 4, pp. 213-227, 2011.

[2] D. Brambilla et al., "Nanotechnologies for Alzheimer's disease: diagnosis, therapy, and safety issues," Nanomedicine: Nanotechnology, Biology, and Medicine, vol. 7, no. 5, pp. 521-540, 2011.

[3] M. H. Cho et al., "Autophagy in microglia degrades extracellular $\beta$-amyloid fibrils and regulates the NLRP3 inflammasome," Autophagy., vol. 10, no. 10, pp. 1761-1775, 2014.

[4] S. H. Han et al., "Effective screen for amyloid $\beta$ aggregation inhibitor using amyloid $\beta$-conjugated gold nanoparticles, " Int J Nanomedicine, vol. 15, no. 6, pp. 1-12, 2010.

[5] M. J. Kogan et al., "Nanoparticle-Mediated Local and Remote Manipulation of Protein Aggregation, " Nano Lett,. vol. 6, no. 1 pp. 110-115, 2006.

[6] Y. H. Liao et al., "Negatively charged gold nanoparticles inhibit Alzheimer's amyloid- $\beta$ fibrilliation induce fibril dissociation, and mitigate neurotoxicity, "Small, vol. 8, no. 23 pp. 3631-3639, 2012. 
[7] K. Yokoyama and D. R. Welchons. (2007, January 31). "The conjugation of amyloid beta protein on the gold colloidal nanoparticles' surfaces." [Online]. Available: http://iopscience.iop.org/article/10.1088/09574484/18/10/105101/meta. 